\title{
Low-Profile Helical Array Antenna Fed from a Radial Waveguide
}

\author{
Hisamatsu Nakano, Fellow, IEEE, Haruo Takeda, Yorihiro Kitamura, Hiroaki Mimaki, and \\ Junji Yamauchi, Member, IEEE
}

\begin{abstract}
A low-profile array antenna composed of 2-turn $4^{\circ}$ pitch angle helices is designed for a frequency band of $11.7 \mathrm{GHz}$ to $12.0 \mathrm{GHz}$. The feed wire of each helix is inserted into a radial waveguide through a small hole and excited by a traveling wave flowing in the transverse electromagnetic (TEM) mode between the two parallel plates of the waveguide. The measured aperture efficiency shows a maximum value of $77 \%$ for a beam radiated in the normal direction and $69 \%$ for a $30^{\circ}$ beam tilt.
\end{abstract}

\section{INTRODUCTION}

$\mathrm{N}$ EW aspects of a forward-fire mode helical antenna have been revealed [1] by using a numerical technique [2]. The combination of low pitch and a small number of turns leads to a low-profile helix as a radiating element of circular polarization. A 2-turn helix of $4^{\circ}$ pitch angle shows a bandwidth of $12 \%$ for a $3-\mathrm{dB}$ axial-ratio criterion.

This paper is a sequel to a previous paper [1] and describes the application of the 2-turn low-pitch helix to an array antenna fed by a radial waveguide [3], [4]. The motivation to apply the helix to the array antenna comes from the requirement of developing a high-efficiency flat-type antenna for direct reception of the broadcasting satellite TV programs (DBS) in Japan. Tilting the beam from the normal direction of the helical array is discussed and experimental results are presented.

\section{Extremely Low-Profile Helix}

First we specify the configuration of a low-profile helical antenna and describe the applicability to a flat-type array antenna.

The low-profile wire helix mounted on an infinite ground plane, corresponding to the upper plate surface of a radial waveguide of infinite extent, is shown in Fig. 1. The wire connected to the feed point is bent at a height $h$ above the ground plane and extended to the starting point of the helix proper. The bending angle is $90^{\circ}$.

In the numerical analysis, image theory is used and a delta-function generator is assumed at the feed point [1]. The configuration parameters are as follows: pitch angle $\alpha=4^{\circ}$, helical circumference $c=25 \mathrm{~mm}=1 \lambda_{12}$, wire radius $\rho=$

Manuscript received February 21, 1991; revised November 12, 1991. The authors are with the College of Engineering, Hosei University, Koganei, Tokyo 184, Japan.

IEEE Log Number 9106603.
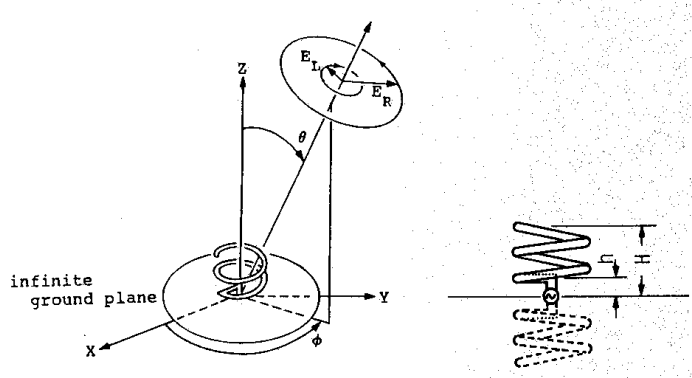

Fig. 1. Configuration of low profile helix

$0.5 \mathrm{~mm}=0.02 \lambda_{12}$, number of helical turns $n=2$, and the bending height $h=1.25 \mathrm{~mm}=0.05 \lambda_{12}$, where $\lambda_{12}(=25$ $\mathrm{mm}$ ) is the free-space wavelength at $12 \mathrm{GHz}$.

In the application to an array antenna, it is desirable that the radiation patterns in any azimuth $\phi$-planes be the same. Fig. 2 shows the calculated radiation patterns in several $\phi$-planes at $12 \mathrm{GHz}$. The radiation field is resolved into two circularly polarized components of $E_{R}$ (principal) and $E_{L}$ (crossed), whose rotational senses are right- and left-handed, respectively. It is confirmed that a similar radiation pattern is obtained in each $\phi$-plane. The half-power beamwidth (HPBW) of the principal polarization component $E_{R}$ is about $70^{\circ}$.

Since the frequency band for direct reception of broadcasting satellite TV programs ranges from 11.7 to $12.0 \mathrm{GHz}$, we also investigate the frequency response of the radiation pattern. Calculations show that the HPBW remains almost constant $\left(70 \pm 1^{\circ}\right)$ in a frequency range of $11.7 \mathrm{GHz}$ to $12 \mathrm{GHz}$. This allows us to approximate the radiation pattern with the function $\cos ^{1.7} \theta$ in volts per meter. The approximated pattern is used in the evaluation of the array pattern based on the pattern multiplication method [5].

\section{Low-Profile Helix Array Fed from a Radial WAVEGUIDE}

\section{A. Configuration of Array Antenna}

Now we array low-profile helices to construct a flat-type antenna [4], as shown in Fig. 3. The transverse electromagnetic (TEM) mode propagates in the radial waveguide provided the spacing of the two parallel plates is sufficiently small compared with the wavelength [3], [6]. For this reason, the spacing $S_{W}$ is chosen to be $7.5 \mathrm{~mm}=0.3 \lambda_{12}$. 


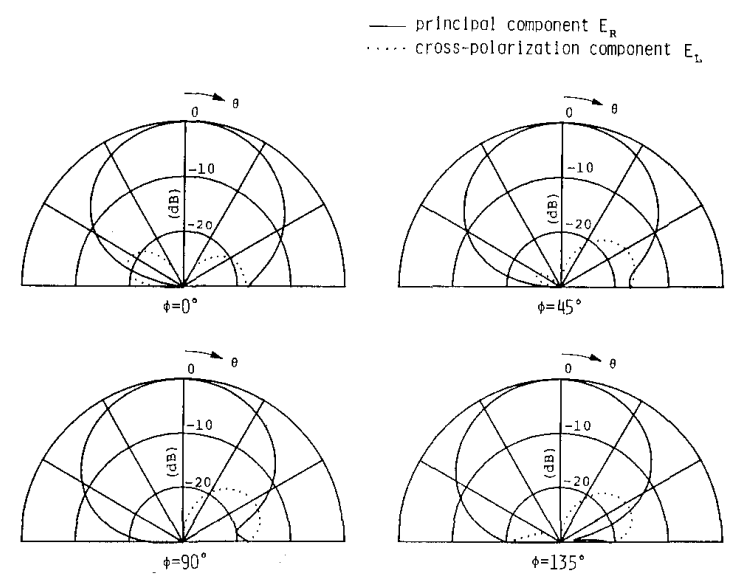

Fig. 2. Radiation pattern of low profile helix at $11.85 \mathrm{GHz}\left(\alpha=4^{\circ}\right.$, $n=2$ ).
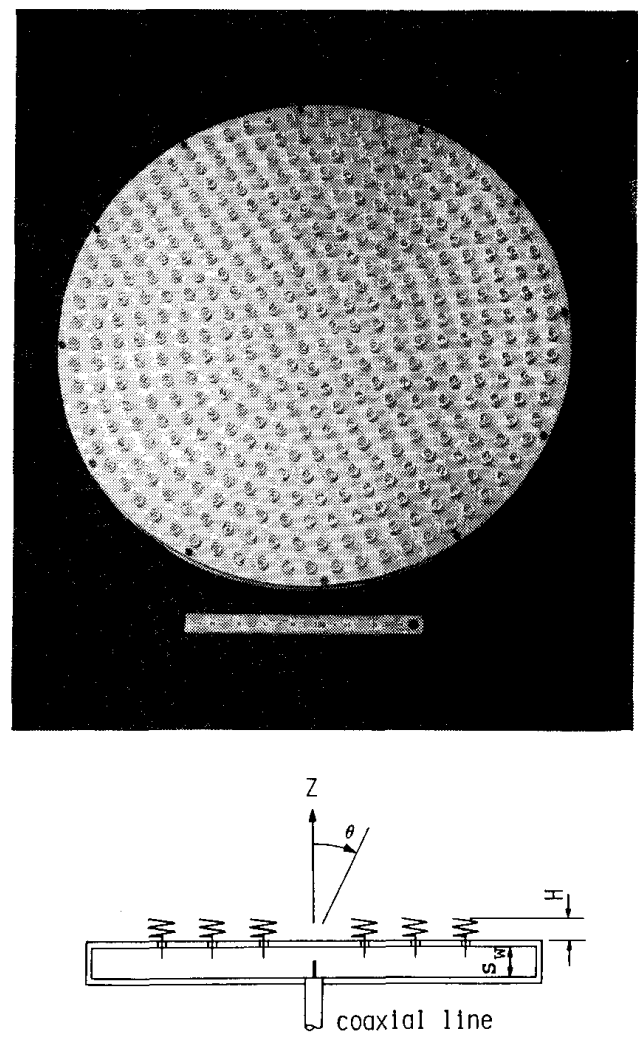

Fig. 3. Flat type antenna consisting of low profile helices.

The feed wire of each helix is inserted into the radial waveguide through a small hole and excited by a traveling wave flowing from the radial waveguide center (coaxial line) toward the waveguide edge. The radius of the feed wire is the same as that of the helix proper. It should be noted that the axial length $H$ of the helical element above the surface of the radial waveguide is extremely low $(H=4.7 \mathrm{~mm})$. The array antenna has a thickness of $15 \mathrm{~mm}\left(=S_{W}(=7.5 \mathrm{~mm})+\right.$ $H(=4.7 \mathrm{~mm})+$ plate thickness $)$.

\section{B. Determination of Spacing of Helical Elements}

When we install a flat-type antenna, such as a microstrip antenna, on a wall in our home for direct reception from a broadcasting satellite, it is necessary to tilt the radiation beam toward the satellite. Array theory indicates that beam tilt can be realized by adjustment of the excitation phase of each element [5]. In the helical array antenna, the adjustment of the excitation phase can be performed by the mechanical rotation of each helical element around its axis [7], without the need for phase shifters.

As the beam tilt angle is increased, the sidelobes become larger, resulting in a decrease in the array antenna directivity. It is therefore important to suppress the sidelobes. This can be achieved by proper spacing of the helical elements.

The spacing is determined, taking the directivity in the direction of $\left(\theta_{0}, \phi_{0}\right)$ into account. The directivity of an $N$-element circular array antenna, shown in Fig. 4(a), can be expressed as [10]

$$
D\left(\theta_{0}, \phi_{0}\right)=\frac{4 \pi\left|F\left(\theta_{0}, \phi_{0}\right)\right|^{2}}{\int_{0}^{2 \pi} d \phi \int_{0}^{\pi}|F(\theta, \phi)|^{2} \sin \theta d \theta}
$$

in which $F(\theta, \phi)$ is the field pattern of the array antenna, and is given as

$$
F(\theta, \phi)=E_{0}(\theta, \phi) \sum_{n=1}^{N} I_{n} e^{j\left[k \rho_{n} \sin \theta \cos \left(\phi-\phi_{n}\right)+\delta_{n}\right]}
$$

where $E_{0}(\theta, \phi)$ is the pattern of the single element, $\rho_{n}$ is the distance from the origin to the $n$th element, and $k$ is the wavenumber. $I_{n}$ and $\delta_{n}$ are, respectively, the excitation amplitude and phase of the $n$th element. For the case of a tilted beam in the $\left(\theta_{0}, \phi_{0}\right)$ direction, we set $\delta_{n}$ to

$$
\delta_{n}=-k \rho_{n} \sin \theta_{0} \cos \left(\phi_{0}-\phi_{n}\right) .
$$

Equation (1) (or (6) in Appendix I) can be calculated by numerical integration, where $E_{0}$ is approximated by the principal component $E_{R}$ (the cross-polarized component $E_{L}$ is neglected).

Fig. 4(b) shows the calculated directivity of a helical array antenna for various spacings of the helical elements as a function of the beam tilt angle from the $Z$-axis. The testing frequency is chosen to be $11.85 \mathrm{GHz}$. In the calculation, the diameter of the helical array antenna, $2 \rho_{\max }$, is kept constant $(38.5 \mathrm{~cm})$, and the helical array antenna is assumed to have a uniform amplitude distribution in its excitation $\left(I_{1}=I_{2}=\right.$ $\left.\cdots=I_{N}\right)$. It is also assumed that each helical element has a radiation pattern of $E_{R}=\cos ^{1 \cdot 7} \theta$. Based on the results shown in Fig. 4(b), a helical array antenna consisting of 396 elements is designed with a circumferential spacing of $S_{\text {cir }}=$ $1.83 \mathrm{~cm}$ and a radial spacing $S_{\text {rad }}=1.75 \mathrm{~cm}$.

\section{Determination of Insertion Length of Feed Wire}

To easily design the array antenna, the field distribution in the radial waveguide should consist of only a forward travel- 


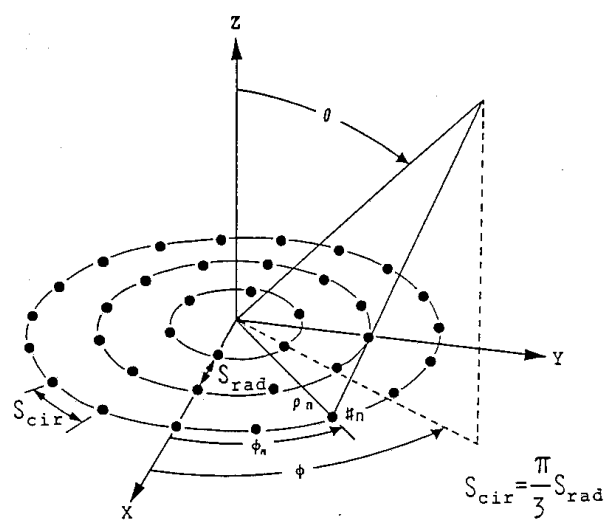

(a)

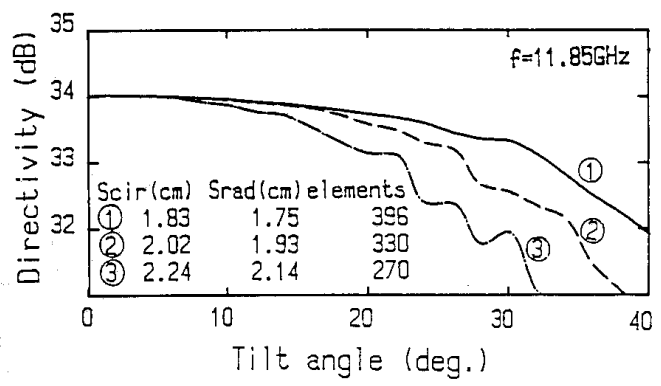

(b)

Fig. 4. Calculated directivity versus beam tilt angle.

ing wave flowing from the waveguide center toward the waveguide edge. This means that we have to eliminate a backward traveling wave reflected from the waveguide edge. In the ideal situation, the input power completely couples into the feed wires, i.e., no energy exists at the waveguide edge. In practice, however, some portion of the forward traveling wave appears at the waveguide edge and becomes a backward traveling wave. To minimize the backward traveling wave, we insert the feed wires of the outermost helices at a distance of a quarter-wavelength from the waveguide edge, where the standing wave is maximum. The backward traveling wave can be absorbed by the outermost helices and becomes negligible over the waveguide except near the edge.

In addition, we should notice the fact that the forward traveling wave in the waveguide decays as it progresses toward the edge. Therefore, to maintain a uniform amplitude distribution (or a uniform power distribution) over the array surface, we should gradually lengthen the feed wires as the radial distance increases.

On the basis of the above-mentioned considerations, we performed several preliminary experiments, in which the insertion length was changed in $0.25 \mathrm{~mm}$ increments. As a result, the insertion lengths are selected to be $2,2.5,3,3$, $3.5,3.5,3.75,4.5,4.5,5.5$, and $7 \mathrm{~mm}$, from the center to the edge.

Fig. 5 shows the measured result of the relative power over the array surface at $11.85 \mathrm{GHz}$. The power is represented by the radius of the black dot. It is found that the array

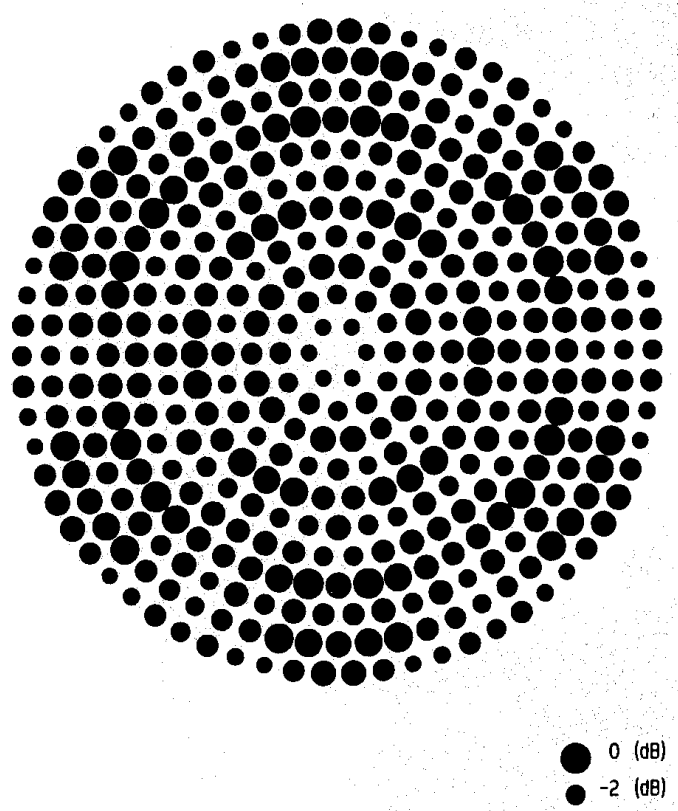

Fig. 5. Measured relative power at 11:85 GHz.

is almost uniformly excited. The difference in the power among the helical elements is within $3 \mathrm{~dB}$. The data are obtained by the technique developed by Mano and Katagi [8], a summary of which is given in Appendix II.

Impedance matching to the coaxial line located in the center of the lower plate of the waveguide is made by proper insertion of the inner conductor of the coaxial line into the waveguide. Good impedance matching is shown in Fig. 6, where the measured return-loss is less than $-18 \mathrm{~dB}$ in the frequency band from $11.7 \mathrm{GHz}$ to $12 \mathrm{GHz}$.

\section{Radiation Characteristics of the Helical Array}

The radiation beam can be tilted by realizing the required phase condition, as shown in (3). The amplitude distribution remains almost unchanged regardless of the helix rotation, so that there is no need to readjust the insertion length of the feed wire. Since the relative phase of each helix can be determined, as described in Appendix II, the only thing we have to do is to rotate each helix around its axis according to the phase requirement determined by (3).

Fig. 7 shows the measured gain at a frequency of 11.85 $\mathrm{GHz}$, as a function of the beam tilt angle. The gain at a beam tilt angle of $30^{\circ}$ is found to be only $1.1 \mathrm{~dB}$ lower than that without the beam tilt (normal beam). The aperture efficiency is calculated to be between $69 \%$ and $77 \%$ over a beam tilt angle of $0-30^{\circ}$. The aperture efficiency, taking account of the beam tilt angle $\theta_{0}$, is evaluated by $\eta=G_{\text {mes }} /$ $\left\{\left(\pi D_{\text {array }} / \lambda\right)^{2} \cos \theta_{0}\right\}$, where $G_{\text {mes }}$ is the measured gain and $D_{\text {array }}$ is the physical array antenna diameter. $D_{\text {array }}$ is 40.25 $\mathrm{cm}\left[=2\left(\rho_{\text {max }}+S_{\text {rad }} / 2\right)=2\left\{\left(11\right.\right.\right.$ rings with $S_{\text {rad }}=1.75$ $\mathrm{cm})+\left(\right.$ edge space of $\left.\left.\left.S_{\text {rad }} / 2\right)\right\}=38.5 \mathrm{~cm}+1.75 \mathrm{~cm}\right]$. It should be noted that conventional microstrip antenna arrays have never attained such high aperture efficiencies. 


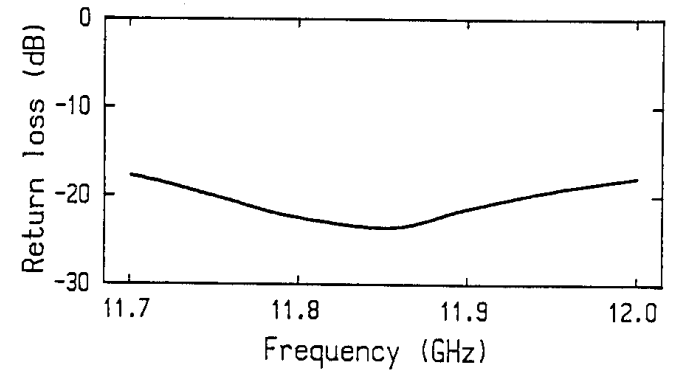

Fig. 6. Measured return loss.
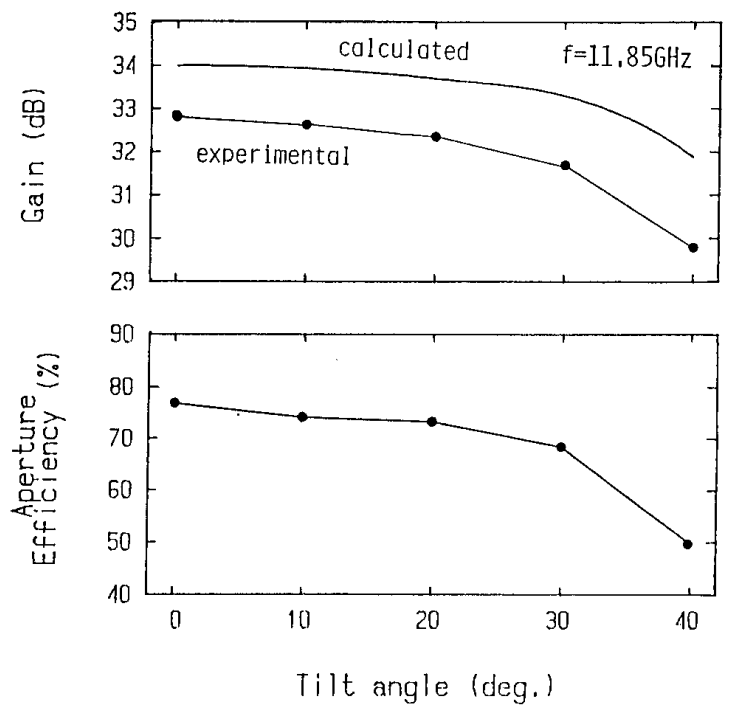

Fig. 7. Measured gain and aperture efficiency at $11.85 \mathrm{GHz}$.

The measured radiation patterns at $11.85 \mathrm{GHz}$ are presented in Fig. 8 for beam tilt angles of 0 and $30^{\circ}$. The half-power beamwidths are $3.7^{\circ}$ and $4.2^{\circ}$, respectively, and the first sidelobe levels are $-18 \mathrm{~dB}$ and $-17 \mathrm{~dB}$. The axial ratio shows a value of less than $1 \mathrm{~dB}$ for each tilt angle. For comparison, the patterns calculated from (2) are also plotted by dotted lines in Fig. 8. We can find good agreement between the calculated and experimental results. Fig. 9 shows the measured frequency responses of the gain and aperture efficiency for these two tilt angles. For a $30^{\circ}$ beam tilt, the aperture efficiency varies from $54 \%$ to $69 \%$, while for a normal beam $\left(0^{\circ}\right.$ beam tilt), the aperture efficiency changes from $66 \%$ to $77 \%$.

It is noted that the beam direction is a function of frequency. Calculations show that the overall beam-shift is $0.4^{\circ}$ over a frequency band from $11.7 \mathrm{GHz}$ to $12 \mathrm{GHz}$ for a $30^{\circ}$ beam tilt. This leads to only a slight degradation of $0.12 \mathrm{~dB}$ in the gain.

Finally, we refer to the mutual coupling among helices. In [9], we describe a mutual coupling among low-profile helices. The mutual coupling changes not only by the spacing but also by the rotation angle.

If each helix radiates without the mutual coupling and only a forward traveling wave flows in the waveguide, the phase

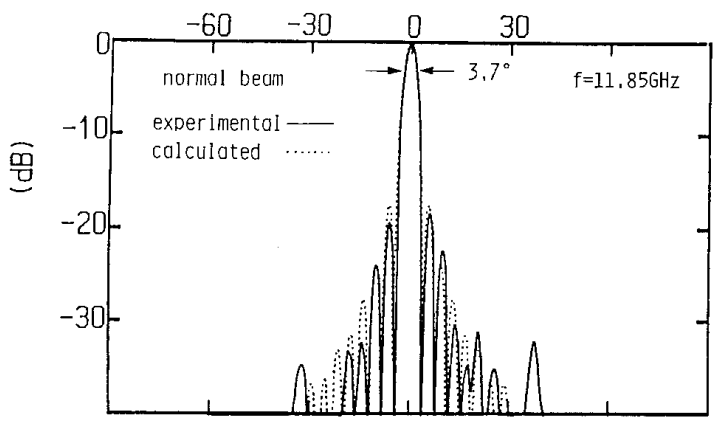

(a)

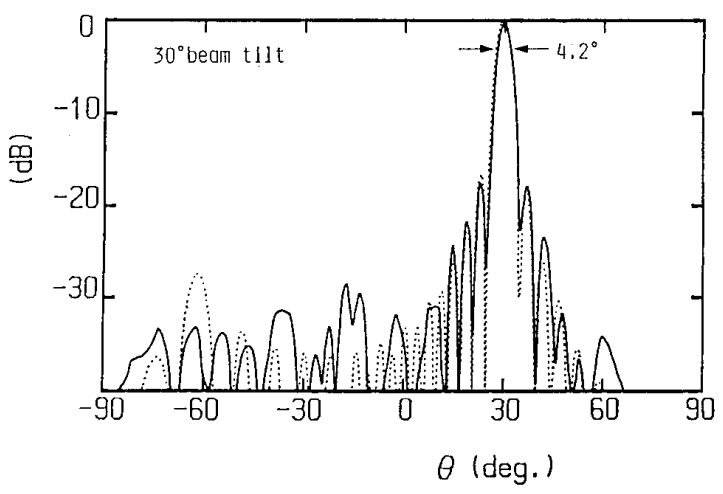

(b)

Fig. 8. Measured radiation patterns at $11.85 \mathrm{GHz}$.
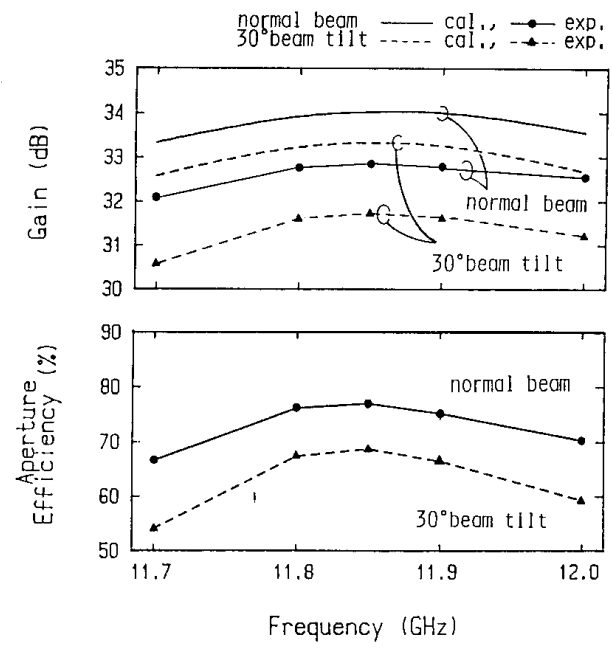

Fig. 9. Measured frequency responses of gain and aperture efficiency.

corresponding to the rotation angle of each helix is determined by the electrical distance that the traveling wave passes through in the radial direction. In practical design, however, we are forced to adjust the rotation angle, due to the presence of mutual coupling. Repetition of the technique, taking account of the mutual coupling among helices, described in Appendix II, determines the amplitude and phase of each helix. 


\section{CONCLUSION}

An extremely low-profile helical array antenna fed from a radial waveguide has been designed for a frequency band of 11.7 to $12.0 \mathrm{GHz}$. A 2-turn helix of $4^{\circ}$ pitch angle is employed as an array element. The calculation shows how proper spacing of the helical elements serves to reduce the deterioration in the directivity of the helical array antenna, when the radiation beam is tilted from the normal direction of the array plane. The measured aperture efficiency shows a maximum value of $77 \%$ for a beam radiated in the normal direction and $69 \%$ for a $30^{\circ}$ beam tilt.

\section{APPENDIX I}

Using (3), (2) is written as

$$
F(\theta, \phi)=E_{0}(\theta, \phi) \sum_{n=1}^{N} I_{n} e^{j k \rho_{n} a_{n}\left(\theta, \phi, \theta_{0}, \phi_{0}, \phi_{n}\right)}
$$

where

$$
\begin{aligned}
a_{n}\left(\theta, \phi, \theta_{0}, \phi_{0}, \phi_{n}\right)=\sin \theta & \cos \left(\phi-\phi_{n}\right) \\
& -\sin \theta_{0} \cos \left(\phi_{0}-\phi_{n}\right) .
\end{aligned}
$$

Consequently, (1) can be written as

$$
\begin{aligned}
D\left(\theta_{0}, \phi_{0}\right) & =D_{\text {tilt }} \\
& =\frac{\left|E_{0}\left(\theta_{0}, \phi_{0}\right) \sum_{n=1}^{N} I_{n}\right|^{2}}{\sum_{n=1}^{N} \sum_{n^{\prime}=1}^{N} b_{n n^{\prime}}\left(\theta, \phi, \theta_{0}, \phi_{0}\right) I_{n} I_{n^{\prime}}^{*}}
\end{aligned}
$$

where the asterisk denotes complex conjugate quantity, and $b_{n n}$ is the mutual radiation resistance given by

$$
\begin{aligned}
& b_{n n^{\prime}}\left(\theta, \phi, \theta_{0}, \phi_{0}\right)=\frac{1}{4 \pi} \int_{0}^{2 \pi} \int_{0}^{\pi} \\
& \cdot\left|E_{0}(\theta, \phi)\right|^{2} e^{j k\left(\rho_{n} a_{n}-\rho_{n}^{\prime} a_{n}^{\prime}\right)} \sin \theta d \theta d \phi .
\end{aligned}
$$

Similarly, the directivity in the case of a normal beam $\left(\theta_{0}=0, \phi_{0}=0\right)$ is

$$
\begin{aligned}
& D\left(\theta_{0}=0, \phi_{0}=0\right)=D_{\text {norm }} \\
& \quad=\frac{\left|E_{0}\left(\theta_{0}=0, \phi_{0}=0\right) \sum_{n=1}^{N} I_{n}\right|^{2}}{\sum_{n=1}^{N} \sum_{n^{\prime}=1}^{N} b_{n n^{\prime}} \cdot\left(\theta, \phi, \theta_{0}=0, \phi_{0}=0\right) I_{n} I_{n^{\prime}}^{*}} .
\end{aligned}
$$

The relation between $D_{\text {tilt }}$ and $D_{\text {norm }}$ is given as

$$
\begin{aligned}
\frac{D_{\text {tilt }}}{D_{\text {norm }}} & =\frac{\left|E_{0}\left(\theta_{0}, \phi_{0}\right)\right|^{2}}{\left|E_{0}\left(\theta_{0}=0, \phi_{0}=0\right)\right|^{2}} \\
& \frac{\sum_{n=1}^{N} \sum_{n^{\prime}=1}^{N} b_{n n^{\prime}}\left(\theta, \phi, \theta_{0}=0, \phi_{0}=0\right) I_{n} I_{n^{\prime}}^{*}}{\sum_{n=1}^{N} \sum_{n^{\prime}=1}^{N} b_{n n^{\prime}}\left(\theta, \phi, \theta_{0}, \phi_{0}\right) I_{n} I_{n^{\prime}}^{*}} .
\end{aligned}
$$

We note that the ratio of $D_{\text {tilt }}$ to $D_{\text {norm }}$ cannot be determined by only the element pattern $E_{0}(\theta, \phi)$, due to the existence of the mutual radiation resistance $b_{n n^{\prime}}$.

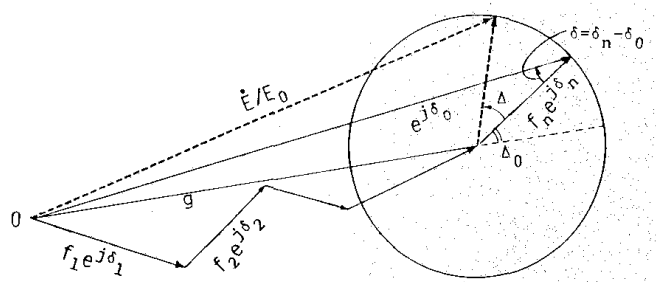

Fig. 10. Vectors of radiation fields from array antenna elements

APPENDIX II

Measurement of the Amplitude and Phase of an Array Element [8]

The total electric field vector $E_{0} \exp \left(j \delta_{0}\right)$ of an array antenna consisting of $N$ elements (1, $2, \cdots, m, \cdots, n, \cdots, N)$ can be expressed by $\sum_{m=1}^{N} E_{m}$ $\exp \left(j \delta_{m}\right)$, where $E_{0}$ and $\delta_{0}$ are the amplitude and the phase of the total electric field for the initial arrangement, and $E_{m}$ and $\delta_{m}$ are those for the $m$ th element, respectively. When we change the phase of the $n$th element by $\Delta$, the total field can be written as

$$
\dot{E}=\left(E_{0} e^{j \delta_{0}}-E_{n} e^{j \delta_{n}}\right)+E_{n} e^{j\left(\delta_{n}+\Delta\right)}
$$

The relative power of the total field can be written as

$$
|\dot{E}|^{2} / E_{0}^{2}=g^{2}+f_{n}^{2}+2 f_{n} g \cos \left(\Delta+\Delta_{0}\right)
$$

where $f_{n}$ is the normalized magnitude of the electric field vector of the $n$th element,

$$
f_{n}=E_{n} / E_{0}
$$

and $g$ is the normalized magnitude of the electric field vector without the contribution of the $n$th element,

$$
g=\left|\sum_{\substack{m=1 \\(m \neq n)}}^{N} f_{m} e^{j \delta_{m}}\right|=\sqrt{\left(\cos \delta-f_{n}\right)^{2}+\sin ^{2} \delta}
$$

in which

$$
\begin{aligned}
\delta & =\delta_{n}-\delta_{0} \\
\tan \Delta_{0} & =\sin \delta /\left(\cos \delta-f_{n}\right)
\end{aligned}
$$

Equation (11) reveals that maximum relative power is obtained for $\Delta=-\Delta_{0}$. This fact is explicitly shown in Fig. 10 . Now, we define the ratio of the maximum and minimum values of (11) as

$$
r^{2}=\left(g+f_{n}\right)^{2} /\left(g-f_{n}\right)^{2}
$$

For the condition $f_{n}<g$, the relative amplitude $f_{n}$ and phase $\delta$ of the $n$th element can be given as

$$
f_{n}=\frac{\Gamma}{\sqrt{1+2 \Gamma \cos \Delta_{0}+\Gamma^{2}}}
$$


and

$$
\delta=\tan ^{-1}\left(\frac{\sin \Delta_{0}}{\cos \Delta_{0}+\Gamma}\right)
$$

respectively, where

$$
\Gamma=f_{n} / g=(r-1) /(r+1) .
$$

In summary, we measure the variation of the relative power $|\dot{E}|^{2} / E_{0}^{2}$ while rotating the $n$th element, and then obtain $-\Delta_{0}$ and $r$. The relative amplitude and phase can be evaluated using (17) and (18), respectively.

\section{REFERENCES}

[1] H. Nakano, H. Takeda, T. Honma, H. Mimaki, and J. Yamauchi, "Extremely low-profile helix radiating a circularly polarized wave," IEEE Trans. Antennas Propagat., vol. 39, pp. 754-757, 1991.

[2] H. Nakano, Helical and Spiral Antennas, Res. Studies Press. New York: Wiley, 1987.

[3] F. J. Goebels and K. C. Kelly, "Arbitrary polarization from annular slot planar antennas," IRE Trans. Antennas Propagat., vol. AP-9, pp. 342-349, 1961.

[4] H. Nakano, H. Mimaki, H. Takeda, and J. Yamauchi, "A low-profile helix radiating a circularly polarized wave," in Proc. ISAP '89, 1989, pp. 501-504.

[5] A. Balanis, Antenna Theory. New York: Harper \& Row, 1982, ch. 6 .

[6] N. Marcuvitz, Waveguide Handbook. New York: McGraw-Hill,

[7] H. Nakano, N. Asaka, and J. Yamauchi, "Short helical antenna array fed from a waveguide," IEEE Trans. Antennas Propagat., vol. AP-32, pp. 836-840, 1984.

[8] S. Mano and T Katagi, "A method of measuring amplitude and phase of each radiating element of a phased array antenna," Trans. IECE Japan, vol. J-65-B, pp. 555-560, 1982.

[9] H. Nakano, N. Asaka, and J. Yamauchi, "Radiation characteristics of short helical antenna and its mutual coupling," Electron. Lett., vol. 20, pp. 202-204, 1984.

[10] J. D. Kraus, Antennas. New York: McGraw-Hill, 1950, p. 542.

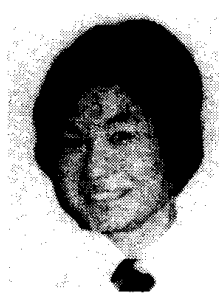

Hisamatsu Nakano (M'75-SM'87-F'92) was born in Tharaki, Japan, on April 13, 1945. He received the B.E., M.E., and Dr.E. degrees in electrical engineering from Hosei University, Tokyo, Japan, in 1968, 1970, and 1974, respectively.

Since 1973, he has been a member of the faculty of Hosei University, where he is now a Professor of electrical engineering. His research topics are numerical methods for microwave antennas and scattering problems.

He was a Visiting Associate Professor at Syracuse University (May-September 1981), where he worked on numerical analysis of electromagnetic coupling between wires and slots; a visiting professor at the University of Manitoba (March-September 1986) working on analysis of microstrip antennas; and a Visiting Professor at the University of California, Los Angeles (September 1986 to March 1987) working on microstrip line antenna analysis. He has published more than 100 refereed papers on antenna problems, and is the author of a book, Helical and Spiral Antennas (Research Studies Press, Wiley).
Dr. Nakano was a recipient of an international scientific exchange award from the Natural Sciences and Engineering Research Council of Canada. In 1987 he received the Best Paper Award of the IEE Fifth International Conference on Antennas and Propagation. He is an Associate Editor of the IEEE Antennas and Propagation Magazine, and a member of the Institute of Electronics, Information and Communication Engineers of Japan.

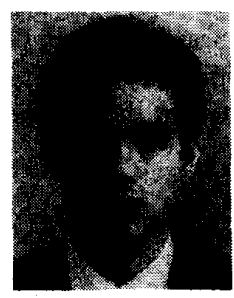

Haruo Takeda was born in Tokyo, Japan, on February 15, 1966. He received the B.E. and M.E. degrees from Hosei University, Tokyo, Japan, in 1988 and 1990, respectively.

He joined the NEC Co. Ltd., Tokyo, in 1990.

Mr. Takeda is a member of the Institute of Electronics, Information and Communication Engineers of Japan.

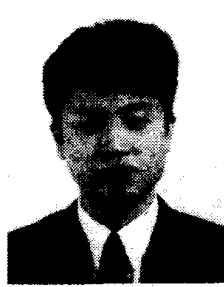

Yorihiro Kitamura was born in Kanagawa, Japan, on January 21,1968 . He received the B.E. degree from Hosei University, Tokyo, Japan, in 1991.

He is now working toward the M.E. degree.

Mr. Kitamura is a member of the Institute of Electronics, Information and Communication Engineers of Japan.

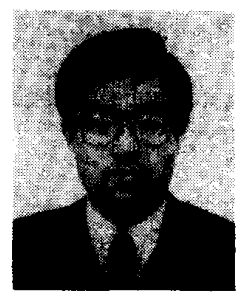

Hiroaki Mimaki was born in Yamaguchi, Japan, on December 7,1952 . He received the B.E. degree in electronic engineering from Tokyo Denki University, Tokyo, Japan, in 1976 and the M.E. degree in electrical engineering from Hosei University, Tokyo, Japan, in 1981

He is currently an Assistant at Hosei University. His research interests are in thin wire antennas.

Mr. Mimaki is a member of the Institute of Electronics, Information and Communication Engineers of Japan.

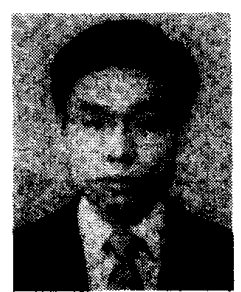

Junji Yamauchi (M'85) was born in Nagoya, Japan, on August 23, 1953. He received the B.E. M.E., and Dr.E. degrees from Hosei University, Tokyo, Japan, in 1976, 1978, and 1982, respectively.

From 1984 to 1988 , he served as a Lecturer at the Electrical Engineering Department of Tokyo Metropolitan Technical College. Since 1988, he has been a member of the Faculty of Hosei University, where he is an Associate Professor of Electrical Engineering. His research interests include circularly polarized antennas and waveguides.

Dr. Yamauchi is a member of the Optical Society of America, and the Institute of Electronics, Information and Communication Engineers of Japan. 